\title{
Ärztliche Fortbildung - continuing medical education (CME)
}

\author{
Continuing Medical Education (CME)
}

\author{
Autoren \\ M. Wildner ${ }^{1}$, M. Mohrmann \\ Institute \\ ${ }^{1}$ Bayerisches Landesamt für Gesundheit und Lebensmittelsicherheit, München \\ ${ }_{2}^{2}$ Medizinischer Dienst der Krankenversicherung, Lahr
}

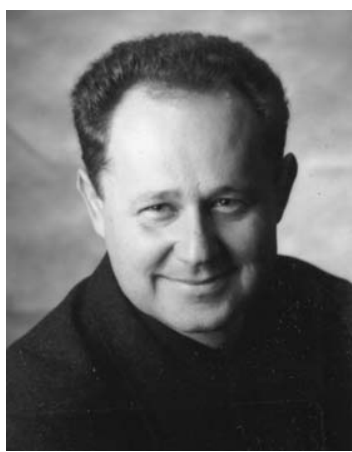

Prof. Dr. Manfred Wildner

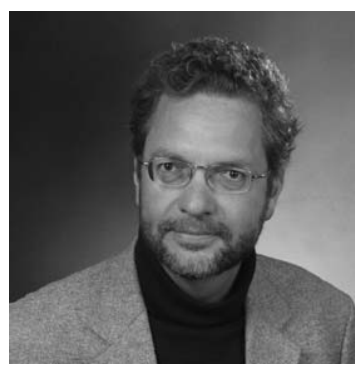

PD Dr. Matthias Mohrmann

Bibliografie

DOI $10.1055 / \mathrm{s}-0028-1082309$

Gesundheitswesen 2008;

70: $379-380$

(c) Georg Thieme Verlag KG Stuttgart · New York

ISSN 0941-3790

Korrespondenzadressen

Prof. Dr. M. Wildner, MPH

Bay. Landesamt für Gesundheit und Lebensmittelsicherheit Veterinärstr. 2

85762 Oberschleissheim manfred.wildner@Igl.bayern.de PD Dr. Matthias Mohrmann Leitender Arzt

Medizinischer Dienst der

Krankenversicherung

Baden-Württemberg

Ahornweg 2

77933 Lahr

matthias.mohrmann@mdkbw.de
„Deutschlands wichtigste Ressourcen sind der Ideenreichtum und die Talente seiner Menschen. [...] die Liste der Herausforderungen ist lang: Fragen des Klimawandels und der Globalisierung verlangen genauso nach zukunftsfähigen Lösungen wie Migration und demographischer Wandel. Um diese Aufgaben zu meistern, müssen wir Bildung, Wissenschaft, Forschung und Innovation in unserem Land stärken “ [1]. Nach diesen Gedanken des Vorworts nennt der Bundesbericht Forschung und Innovation 2008 Gesundheit als ersten von vier „Leitmärkten“ neben Sicherheit, Klima- und Ressourcenschutz und Mobilität. Die thematischen Schwerpunkte? Forschung zum Gesundheitswesen, Verbesserung der Qualität und Versorgung in der Gesetzlichen Krankenversicherung und in der sozialen Pflegeversicherung, Begleitforschung zur Umsetzung von Reformen und Gesetzen und Prävention und Gesundheitsförderung sind einige davon [1,S. 235ff]. Diese Schwerpunktsetzung unterstreicht die seit Langem gegebenen Entwicklungsdynamiken im $\mathrm{Ge}$ sundheitswesen - seien es technologische Neuerungen (Arzneimittel inbegriffen), seien es betriebswirtschaftlich-organisatorische Reformen auf Anbieterseite oder seien es systemorientierte Weiterentwicklungen einschließlich Leitlinien und evidenzbasierter Medizin.

Die ärztliche (Selbst-)Verpflichtung zur kontinuierlichen Fortbildung korrespondiert zu dieser Entwicklung, teilweise als ihre Folge, teilweise auch als ihr Antrieb. In $\S 4$ der (Muster-)Berufsordnung ist die Fortbildungsverpflichtung berufsrechtlich festgelegt. Seit dem 1. Januar 2004 (GKV-Modernisierungsgesetz - GMG) gilt eine mit finanziellen Rückbehalten bewehrte fünfjährliche Nachweispflicht der ärztlichen Fortbildung für Vertragsärzte ( $§ 95 d$ SGB V) wie auch für Fachärzte im Krankenhaus (§137 Abs. 1 Nr. 2 SGB V). Zeitlich vorausgehend wurde von der Europäischen Union 1993 eine Resolution zur ärztlichen Fortbildung verabschiedet: „Continuing medical education should remain an ethical obligation subject to the disciplinary authority of the profession itself. Continuing medical education should be both an individual and also a collective obligation of the profession [...]“ (Deutsch: Ärztliche Fortbildung sollte als ethische Verpflichtung in der Zuständigkeit der ärztlichen Profession liegen. Ärztliche Fortbildung sollte sowohl eine individuelle als auch eine kollektive professionelle Verpflichtung sein [...]) [2]. Von der Europäischen Union der Fachärzte (UEMS) wurde dieser Anspruch aufgegriffen und in der "Charter on continuing medical education of medical specialists in the European Union“" von 1994 weiter konkretisiert [3]. Artikel 5 dieser Charter legt fest, dass zu diesem Zweck ein Kreditpunkte-System von der ärztlichen Selbstverwaltung entwickelt werden soll.

Für Deutschland wurde auf dem 107. Deutschen Ärztetag 2004 in Bremen eine entsprechende Musterfortbildungssatzung entwickelt und durch Empfehlungen der Bundesärztekammer zur ärztlichen Fortbildung ergänzt [4,5]. Ziel der Fortbildung der Ärzte und der Ärztinnen sind „der Erhalt und die dauerhafte Aktualisierung der fachlichen Kompetenz". Der Inhalt der Fortbildung wird wie folgt beschrieben: „Durch die Fortbildung soll unter Berücksichtigung der wissenschaftlichen Erkenntnisse und neuer medizinischer Verfahren das zum Erhalt und zur Fortentwicklung der Kompetenz notwendige Wissen in der Medizin und der medizinischen Technologie vermittelt werden. Fortbildung soll sowohl fachspezifische als auch interdisziplinäre und fachübergreifende Kenntnisse und die Einübung von klinisch-praktischen Fähigkeiten umfassen. Die Fortbildung soll sich dabei auf alle medizinischen Fachrichtungen in ausgewogener Weise erstrecken. Ärztliche Fortbildung umfasst auch die Verbesserung kommunikativer und sozialer Kompetenzen. Die ärztliche Fortbildung schließt außerdem Methoden der Qualitätssicherung, des Qualitätsmanagements und der evidenzbasierten Medizin ein. Bundeseinheitliche Vorgaben 
zum angemessenen Umfang der Fortbildung sind zu beachten“ [4].

Mediengestütztes Eigenstudium wird ausdrücklich als geeignete Fortbildungsmethode genannt. Die Fortbildungsmaßnahmen werden mit Punkten bewertet. Grundeinheit ist eine 45-minütige Fortbildungseinheit. Damit ist die Fortbildungsverpflichtung auch ein Fortbildungsauftrag an die wissenschaftlichen Fachzeitschriften. Das „Deutsche Ärzteblatt“ berichtet von durchschnittlich 19000 Teilnehmern an den dort veröffentlichten CME-Artikeln [6]. Etwa ein Viertel der Ärzteschaft beteiligte sich im dreieinhalbjährigen Auswertezeitraum ein- oder mehrmals an diesem Fortbildungsangebot. Im Durchschnitt wurden 95\% der gestellten Fragen von den Lesern richtig beantwortet - CME-Evaluationen sind eher Lesekontrollen, weniger Prüfungen.

Dass die Formulierung der zugehörigen Multiple-Choice-Fragen problematisch sein kann, haben Studien in den USA und in Deutschland gezeigt $[7,8]$. Die Effektivität ärztlicher Fortbildung scheint bei interaktiven Programmen bzw. bei einem Methoden-Mix am höchsten zu sein [9]. Allgemein ist darauf zu achten, dass Ärztliche Fortbildung unabhängig von kommerziellen Interessen ist und ein ausgewogener Überblick über dem jeweiligen Wissensstand entsprechende diagnostische und therapeutische Alternativen vermittelt wird [4].

Ab diesem Heft wird auch „Das Gesundheitswesen“ mit CMEKreditpunkten versehene Fortbildungsartikel anbieten, zunächst in zweimonatigen Abständen. Diese sind bei der Landesärztekammer Nordrhein zertifiziert (siehe auch http://cme.thieme.de > über CME). Die Zuständigkeit in der Schriftleitung liegt bei Matthias Mohrmann. Die Autoren der einzelnen Beiträge sowie die zitierten Referenzen werden entsprechend den Regeln wissenschaftlicher Arbeiten jeweils genannt, die CME-Fortbildungsartikel werden zur Qualitätssicherung alle einem Experten-Review unterzogen. Die Erfolgskontrolle erfolgt über MultipleChoice-Fragen, die unter Einhaltung der datenschutzrechtlichen Vorgaben vom Thieme-Verlag ausgewertet werden. Wir möchten Sie bei dieser Gelegenheit einladen, der Schriftleitung CME-Fortbildungsartikel für „Das Gesundheitswesen“ einzureichen oder aber auch Themenvorschläge für solche Artikel zu machen.

Bei der thematischen Auswahl wird die Förderung praxisrelevanter, nachhaltiger und gleichzeitig zukunftsorientierter Kompetenzen bedacht. Neben Verständlichkeit, Relevanz und Aktualität erfolgt ggf. auch eine kritische Wertung im Kontext des Themenfeldes, auch unter Beachtung einschlägiger ethischer Grundsätze. So sollen das Wissen, die Befähigungen und die Ein- stellungen geschaffen werden, die unseren Kenntnisstand erweitern, unser praktisches Arbeiten prägen und Orientierung geben können. Europa hat sich zum Ziel gesetzt, bis 2010 zum dynamischsten wissensbasierten Wirtschaftsraum zu werden. Damit stehen wir vor der Aufgabe, auf dem Weg in die Wissensgesellschaft auch in unserem Feld voranzugehen und den gemeinsamen Europäischen Lebens- und Innovationsraum mitzugestalten [10]. Und damit die in den Empfehlungen zur ärztlichen Fortbildung gemachte Aussage einzulösen: Berufsbegleitende Aktualisierung des Wissens und kontinuierliche Erweiterung der fachlichen Kompetenz gehören zum ärztlichen Selbstverständnis [5].

Noch eine weitere Neuerung als eine „continuing medical education“ der besonderen Art werden Sie in diesem Heft finden: die Rubrik Das Porträt mit „15 Fragen an...“. Die von Anne Brunner von der Hochschule München zusammengestellten Fragen richten sich in dieser Ausgabe an Jörg Hinrich Hacker, Präsident des Robert Koch-Instituts in Berlin. Diese Interviews werden künftig in lockerer Folge erscheinen.

\section{Literatur}

1 BMBFT. Bundesbericht Forschung und Innovation 2008, S. 3, http:// www.bmbf.de/pub/bufi_2008.pdf, zugegriffen am 30.6.2008

2 Advisory Committee on Medical Training (ACMT) of the European Commission(CP93/96); http://cpme.dyndns.org:591/adopted/cp\%201993 96.pdf, zugegriffen am 30.6.2008

3 UEMS.Charter on continuing medical education of medical specialists in the European Union; http://admin.uems.net/uploadedfiles/174.pdf, zugegriffen am 30.6.2008

4 Bundesärztekammer. Musterfortbildungssatzung; http://www.bundesaerztekammer.de/page.asp?his=1.102.103.160, zugegriffen am 30.6.2008

5 Bundesärztekammer. Empfehlungen der Bundesärztekammer zur ärztlichen Fortbildung. http://www.bundesaerztekammer.de/page.asp? his=1.102.104

6 Baethge C, Marx C. CME im Deutschen Ärzteblatt - eine Auswertung nach dreieinhalb Jahren. Dt Ärzteblatt 2008; 105: 204-206

7 Stagnaro-Green AS, Downing SM. Use of flawed multiple-choice items by the New England Journal of Medicine for continuing medical education. Med Teach. 2006; 28 (6): 566-568

8 Kühne-Eversmann L, Nussbaum C, Reincke M, Fischer MR. CME-Fortbildungsangebote in medizinischen Fachzeitschriften: Strukturqualität der MC-Fragen als Erfolgskontrollen Am Beispiel der Fachzeitschriften Deutsches Ärzteblatt, Deutsche Medizinische Wochenschrift und Der Internist. Med Klin (Munich) 2007; 102 (12): 993-1001

9 Satterlee WG, Eggers RG, Grimes DA. Effective medical education: insights from the cochrane library. Obstet Gynecol Surv 2008; 63 (5): 329-333

10 BMBFT. Bundesbericht Forschung 2004, II http://www.bmbf.de/pub/ bufo2004.pdf, zugegriffen am 30.6.2008 